\title{
450 Millionen Euro Sondermittel für Verhaltenstherapie - Ist die britische Initiative für psychisch Kranke ein Modell für Deutschland?
}

\author{
Winfried Rief \\ Klinische Psychologie und Psychotherapie, Philipps-Universität Marburg, Deutschland
}

\begin{abstract}
Nachdem das englische Gesundheitsministerium vergangenes Jahr die Mittel freigegeben hat, laufen nun die Vorbereitungen zu einem Projekt zur tiefgreifenden Verbesserung der Versorgungssituation psychisch Kranker: Allein in den ersten drei Jahren werden Sondermittel in Höhe von 300 Millionen Englische Pfund (450 Millionen Euro) zur Verfügung gestellt, um effektivere und effizientere Psychotherapie zu realisieren. Psychisch Kranken sollen nicht nur medikamentöse Behandlungen angeboten werden, sie sollen auch breitenwirksam (also nicht nur eine kleine Untergruppe) effektive Psychotherapie erhalten (Projekt «Improving Access to Psychological Therapies», www.iapt.nhs.uk).
\end{abstract}

Wie sieht diese Initiative aus?

Hintergrund dieser Initiative ist, dass die britische Gesundheitsbehörde anerkennt, dass Psychotherapien kurzfristig mindestens so erfolgreich sind wie medikamentöse Behandlungen und langfristig sogar oft besser abschneiden. Dies gilt besonders für die weit verbreiteten Störungsbilder Depressionen und Angsterkrankungen [Hollon et al., 2006]. Während Pharmakotherapie jedoch jedem psychisch Kranken zur Verfügung steht, beträgt die durchschnittliche Wartezeit für einen Psychotherapieplatz in Großbritannien 18 Monate. Mit einer beispiellosen Aktion soll hier Abhilfe geschaffen werden: Innerhalb kürzester Zeit sollen 8000 neue Psychotherapeuten ausgebildet und zugelassen werden, von denen 3600 bereits in den nächsten 3 Jahren in das Gesundheitssystem integriert werden sollen. Die angehenden Therapeuten sollen besonders in der konsequenten Umsetzung von wissenschaftlich fundierten Ansätzen geschult werden. Nach Berechnungen der britischen Gesundheitsbehörde wird diese Erweiterung des Angebots gerade durch fokussierte psychologische Behandlungsansätze kosteneffektiv. Es wird erwartet, dass die Investitionen in das Projekt durch die Reduktion ineffektiver Behandlungen und sekundärer Krankheitsfolgen (Arbeitsunfähigkeit,
Berentungen usw.) deutlich amortisiert werden. Dabei stützt sich die britische Erkenntnis auf eine Vorstudie aus den englischen Gemeinden Newham und Doncaster. In dieser Vorstudie bestätigte sich nicht nur die Kosteneffizienz, sondern es wurde auch belegt, dass sich die Behandlungssituation zusätzlich verbessert, wenn die Patienten direkt zum Psychotherapeuten gehen können. Bisher ist in England eine Zuweisung zum Psychotherapeuten nur über den Hausarzt möglich. Die Vorschaltung des Hausarztes führte jedoch nicht zu einer verbesserten Zuweisung zum Psychotherapeuten, sondern eher zu einer verzögerten Aufnahme einer ambulanten Psychotherapie.

Eine gigantische Aufgabe steht nun vor den Projektleitern um Prof. David Clark und anderen in London. Innerhalb kürzester Zeit müssen Ausbildungsprogramme festgelegt werden, die sicherstellen, dass die angehenden Psychotherapeuten auch wirklich die Kompetenz haben, zeitlich eng umschriebene, hoch effektive Psychotherapien durchzuführen. Nur wenn auch die Behandlungsdauern kurz sind, kann eine Verbesserung der Gesamtversorgungssituation erreicht werden. Diese Erfahrung hat man bereits in Australien gemacht, wo im Jahr 2006 ein Verfahren zum besseren Zugang zu psychologischen Behandlungen eingeführt wurde, bei dem eine Behandlung durch Psychotherapie über 12 Sitzungen pro Kalenderjahr von den Kassen gedeckt wird ('it is recommended that cognitive behaviour therapy be provided'; www.health.gov.au/internet/main/publishing.nsf/Content/COAA 936E6D40C25CCA2572020002E469/\$File/Q\&As.pdf).

Wäre eine solche Maßnahme auch in Deutschland notwendig? Vordergründig erscheint die psychotherapeutische Versorgung von Menschen mit seelischen Erkrankungen in Deutschland viel besser. Bei der Bundespsychotherapeutenkammer sind 30000 approbierte Psychotherapeuten gemeldet, hinzu kommen alle ärztlichen Psychotherapeuten. Dies ist eine sehr

\section{KARGER}

Fax +497614520714

Information@Karger.de

www.karger.com (c) 2008 S. Karger GmbH, Freiburg

Accessible online at:

www.karger.com/ver
Prof. Dr. Winfried Rief

Fachbereich Psychologie

Universität Marburg

Gutenbergstr. 18, 35032 Marburg, Deutschland

Tel. +49 6421 282-3657, Fax -8904

rief@staff.uni-marburg.de 
große Zahl, und die Versorgungsdichte von Psychotherapeuten in Deutschland zählt international zu den besten. Trotzdem ist die Lage aus Sicht der Patienten kaum besser als in Großbritannien: Zwar beträgt die durchschnittliche Wartezeit auf eine Psychotherapie in Deutschland nur 4-8 und nicht 18 Monate wie in Großbritannien; aber auch das ist unerträglich lange, wenn man an einer schweren Angsterkrankung leidet, wenn man verzweifelt ist, wenn Magersucht zu kontinuierlicher Gewichtsabnahme führt, wenn man nicht mehr arbeitsfähig ist oder die Familie auseinanderzubrechen droht. 11,5\% der Menschen mit schweren Depressionen und rund $17 \%$ der Menschen mit Panikstörung in Deutschland gehen $\mathrm{zu}$ einem Psychotherapeuten [Rief et al., 2005]. In anderen Worten: weit über $80 \%$ der Personen mit diesen häufigen und beeinträchtigenden Erkrankungen erhalten keine Psychotherapie, sondern sind hauptsächlich in hausärztlicher und organmedizinisch orientierter fachärztlicher Behandlung. Bei beiden Störungsbildern gibt es Hinweise darauf, dass eine rein pharmakologische Behandlung nicht so langfristige Erfolge erbringt wie eine konsequente und an wissenschaftlichen Kriterien orientierte Psychotherapie [Hollon et al., 2005, 2006]. Bei manchen psychosomatischen Störungen gelten bestimmte Psychotherapieverfahren zwar als Methode erster Wahl, werden jedoch trotzdem kaum eingesetzt [Weise et al., 2007; Nestoriuc et al., 2008].

In Deutschland mitunter emotional diskutiert wird auch die Tatsache, dass die in der Praxis durchgeführte Psychotherapie in den wenigsten Fällen mit dem übereinstimmt, was in wissenschaftlichen Studien als effektiv nachgewiesen wurde. Dies gilt auch für die Verhaltenstherapie, die an sich wissenschaftlich sehr gut fundiert ist. Unter gesundheitsökonomischen Aspekten sind hier Therapieverfahren im Umfang von 5-15 Stunden zu nennen, die bei wissenschaftlichen Studien oft besonders gut abschneiden. Zwar gibt es Störungen, z.B. Borderline-Persönlichkeitsstörungen, bei denen auch nach wissenschaftlichen Studien in aller Regel längere Behandlungsmaßnahmen angezeigt sind. Trotzdem dürften aktuell weit über $90 \%$ der in Deutschland durchgeführten ambulanten Psychotherapien nicht identisch sein mit einem der wis- senschaftlich gut belegten und hocheffizienten Behandlungsverfahren. Bereits die ambulante Kurzzeitpsychotherapie umfasst in Deutschland üblicherweise 5 probatorische plus 25 Einzeltherapiesitzungen; die durchschnittliche Sitzungszahl der häufig realisierten Langzeittherapie lag vor wenigen Jahren bei 70 Stunden [Löcherbach et al., 1999; vgl. Rief et al., 2006]. Natürlich kann man einwenden, dass sich Patienten in der ambulanten Praxis von Patienten unterscheiden, die in wissenschaftlichen Therapiestudien aufgenommen und behandelt werden. Dies trifft jedoch selbst bei positivster Schätzung höchstens auf $30-40 \%$ der Patienten in der ambulanten Versorgung zu, nicht auf über $90 \%$. Es muss daher als Tatsache gelten, dass die meisten ambulanten Psychotherapien in Deutschland zu lange dauern. Dadurch kommen insgesamt weniger Patienten in den Genuss einer ambulanten Psychotherapie. Kürzere, ökonomische Therapien sind selten [vgl. Helbig and Hoyer, 2007] und auch ökonomische Gruppeninterventionen [z.B. Falge-Kern et al., 2007] werden wenig angeboten. Dazu tragen sicherlich auch die Regelungen bei, die die Kassenärztlichen Vereinigungen für die Abrechnung vorgeben: Kein Psychotherapeut wird dafür belohnt, kosteneffektive, zeitlich kondensierte, hocheffiziente Behandlungen durchzuführen. Es gibt kein echtes Anreizsystem für die Umsetzung wissenschaftlich fundierter Kurzzeittherapien.

Damit stellt sich jedoch die Situation in Deutschland nicht anders dar als in Großbritannien: Ein unbefriedigend geringer Anteil der Personen, die eine psychische Erkrankung erleiden, kommt in den Genuss von ambulanter Psychotherapie. Noch schlechter sieht es bei psychosomatischen/verhaltensmedizinischen Problemen aus. Während Deutschland in der Vergangenheit vielleicht manchmal mit etwas Hohn auf das englische Gesundheitssystem geschaut hat, müssen psychisch Kranke in Zukunft gegebenenfalls mit Neid auf die englische Insel schauen. Wer an den psychisch Kranken im Gesundheitssystem spart, spart an der falschen Stelle. Nicht nur aus ökonomischen Gründen, aber auch aus ökonomischen Gründen ist eine adäquate Behandlung zwingend indiziert. Dazu muss sich auch in Deutschland noch vieles ändern.

\section{Literatur}

Falge-Kern A, Schulz H, Fricke S: Ein Gruppentherapieprogramm bei Persönlichkeitsstörungen und unflexiblen Persönlichkeitsstilen: Eine quasiexperimentelle Evaluation. Verhaltenstherapie 2007;17:17-24.

Helbig S, Hoyer J: Hilft wenig viel? Eine Minimalintervention für Patienten während der Wartezeit auf ambulante Verhaltenstherapie. Verhaltenstherapie 2007; 17:109-115.

Hollon SD, DeRubeis RJ, Shelton RC, Amsterdam JD, Salomon RM, O’Reardon JP, Lovett ML, Young PR, Haman KL, Freeman BB, Gallop R: Prevention of relapse following cognitive therapy vs medications in moderate to severe depression. Arch Gen Psychiatry 2005;62:417-422.
Hollon SD, Stewart MO, Strunk D: Enduring effects for cognitive behavior therapy in the treatment of depression and anxiety. Annu Rev Psychol 2006;57: 285-315.

Löcherbach P, Henrich G, Kemmer H, Kinstler HJ, Knopp-Vater M, Rieckmann N, Schneider A, Weber I: Entwicklung und Indikatoren zur Bedarfsermittlung und Angebotsplanung in der ambulanten Psychotherapie - Ergebnisse einer Studie des Zentralinstituts für die Kassenärztliche Versorgung. Verhaltenstherapie und psychosoziale Praxis 1999;31:615-643.
Nestoriuc Y, Rief W, Martin A: Meta-analysis of biofeedback for tension-type headache: efficacy, specificity, and treatment moderators. J Consult Clin Psychol 2008;76:379-396.

Rief W, Exner C, Martin A: Psychotherapie - Ein Lehrbuch. Kapitel 28. Stuttgart, Kohlhammer, 2006.

Rief W, Martin A, Klaiberg A, Brähler E: Specific effects of depression, panic, and somatic symptoms on illness behavior. Psychosom Med 2005;67:596-601.

Weise C, Heinecke K, Rief W: Biofeedback bei chronischem Tinnitus - Behandlungsleitfaden und vorläufige Ergebnisse zu Wirksamkeit und Akzeptanz. Verhaltenstherapie 2007;17:220-230. 